\title{
Contribuição ao inventário dos Musci brasileiros: 2. Phyllodrepaniaceae.
}

\author{
Olga Yano (*)
}

\section{Resumo}

Phyllodrepaniaceae é representada no Brasil, até o presente, por duas espécies: Phyllodrepanium falcifolium (Schwaegr.) Crosby e Mniomalia viridis (Mitt.) C. Muell.; ambas de ocorrência apenas na Amazônia legal. Mniomalia viridis foi coletada no Município de Barra do Garças, Mato Grosso, e Phyllodrepanium falcifolium, no Território Federal de Roraima, constituindo primeira citação para o local.

\section{INTRODUÇÃO}

Phyllodrepaniaceae é constituída por dois gêneros: Phyllodrepanium Crosby e Mniomalia C. Muell. O primeiro era conhecido como Drepariophyllum Schwaegr. desde 1823 até que Crosby (1970) verificou achar-se esse nome pré-ocupado por Drepanophyllum Wibel, 1799, gênero de Umbelliferae. Proposta a modificação do epíteto do gênero, esta provocou automaticamente alteração do nome da família, para Phyllodrepaniaceae, por ser Phyllodrepanium seu gênero-tipo, representado por apenas uma espécie, $P$. falcifolium (Schwaegr.) Crosby.

O gênero Mniomalia foi descrito por Mueller (1874) como pertencente à família Mniaceae. Em 1884, quando Mueller descreveu a espécie Mniomalia naumannii, fez a numeração das espécies novas ocorrentes em Nova Hannovera e Itha Anachoretes, coletadas por Naumann na expedição S.M.S. "Gazelle", sem mencionar a família. Depois o trabalho de Bartram (1949), quando se refere aos musgos de Guatemala, coloca o gênero na família Drepanophyllaceae. Em seguida, o trabalho de Crosby (1970) confirma o gênero Mniomalia pertencente à família em estudo. Apresenta três espécies validamente publicadas: M. naumannii C. Muell., M. semilimbata (Mitt.) C. Muell. e M. viridis (Mitt.) C. Muell., sendo a última americana.
O objetivo do trabalho é contribuir para o inventário da brioflora brasileira, cuja série se iniciou em 1979 com a publicação da família Helicophyllaceae.

\section{MATERIAL E MÉTODOS}

Foram examinadas exsicatas depositadas nos ierbários científicos: "Maria Eneyda P. Kauffmann Fidalgo", Instituto de Botânica, Secretaria da Agricultura e Abastecimento do Estado de São Paulo (SP); Instituto Nacional de Pesquisas da Amazônia (INPA); Instituto de Pesquisa e Experimentação Agropecuária do Norte, Belém (IAN) e Museu Paraense Emílio Goeldi (MG) .

Para cada estrutura, os limites, máximo e mínimo, foram estabelecidos após a tomada de pelo menos 10 medidas, feitas ao acaso.

$\mathrm{Na}$ distribuição geográfica, estão incluídos dados mencionados em literatura ou obtidos de material de herbário.

\section{MAterial ESTUdAdo}

\section{Phyllodrepanium falcifolium (Schwaegr.) Crosby.}

Revue bryol. lichen. 37(2): 346. 1970 (fig. 5-8). Espécime e localidade tipos: J.M.C. Richard $\mathrm{s} / \mathrm{n}$; Hispaniola, Republica Dominicana $(B, G)$.

Basiônimo: Fissidens falcifolium Schwaegr., Spec. Musc., Suppl. 1(2): 9. 1816.

Gametófito simples, verde-castanho, totalmente castanho quarido seco, $17-20 \mathrm{~mm}$ alt.; ramos fiageliformes geralmente presentes no ápice; na base com poucos rizóides, castanhos. Filídios numerosos, complanados, fortemente falcados, assimétricos, 2,0-2,5(3,0) $\mathrm{mm}$ compr.; terço superior irregularmente denteado; costa forte. quase junto do lado côncavo do filídio,

(') - Seção de Briologia e Pteridologia, Instituto de Botânica, Caixa Postal 4005, 01000 São Paulo, SP. 
percurrente à excurrente; células alongadas na margem $65 \mu \mathrm{m}$ compr., $15-20 \mu \mathrm{m}$ larg.; células medianas do filídio rombo-alongadas $50 \mu \mathrm{m}$ compr., $20 \mu \mathrm{m}$ larg. Esporófio não examinado.

\section{DISTRIBUIÇÃo GEOGRÁFICA}

Espécimes em Literatura - Esta espécie apresenta uma distribuição no Brasil, limitada à região amazônica.

Amazonas: região amazônica (Brotherus, 1909 e 1924j, ambos como Drepanophyllum fulvum Rich.); rio Amazonas (Hornschuch (1840). como D. fulvum); rio Negro, monte Cucuí, col. R. Spruce 547 (Spruce, 1867), Mitten (1869) e Mueller (1901), todos como D. fulvum, Crosby (1970)); Cerro de Canapuma, col. R. Spruce 550 (Crosby (1970)). Pará: rio Amazonas, Tauau, perto de Pará, col. R. Spruce 548 e 552 (Mitten, 1869), Mueller (1901) e Spruce (1867), tedos como D. fulvum), perto de Pará (hoje Belém), col. R. Spruce 552 e 553 (Crosby, 1970). Mato Grosso: Aripuanã, Reserva Florestal, col. C. Damião 2433 e 2447 (Lisboa \& Lisboa, 1978)).

Espécimes Examinados: Amazonas: Manaus, Reserva Campina, km 60 on Manaus-Caracaraí road, on a rotten trunk, col. D. Griffin, D.M. Vital \& O. Yano $563 \mathrm{pp}, 1-\mathrm{VIII}-1974$, det. D. Griffin (INPA, FLAS, SP125463); Manaus, EMBRAPA, km 30 on Manaus-Caracaraí road, col. W.R. Buck 1744, 3-XI-1977 (INPA, NY, SP147782); São Gabriel da Cachoeira, igarapé Freitas, sobre pau podre, na mata úmida, col. O. Yano 1977, 17-VII-1979, det. O. Yano (INPA, SP1448597); rio Maués Mirim, mata da terra firme, col. E. de Oliveira 88pp, 4-VI-1957, det. O. Yano (IAN97973). Pará: Beiém, Jaramacaru-Pedras, sobre madeira morta, col. W. A. Egler 547, 8-VI-1957 (MG23174, IAN39010). Território de Roraima: Acampameno do 6 $\mathrm{P}$ BECJundiá, km 328 on Manaus-Caracaraí road, 17-XI-1977 (INPA, NY, SP147790).

Mniomalia viridis (Mitt.) C. Muell.

J. Mus. Godeffroy 3(6): 61. 1874 (fig. 1-4) .

Espécime e localidade tipos: R. Spruce 553; Rio Amazonas, Pará, Brasil (K) .

Basiônimo: Drepanophyllum viride Mitt., J. Linn. Soc. Bot. 12: 318. 1869.
Gametófito verde-escuro, $8-10 \mathrm{~mm}$ alt., ramificado; ramos flageliformes às vezes presen. tes, no ápice; nia base, muitos rizóides castanhos. Filidios numerosos, obliquamente inseridos, ovalado-oblongo, agudo, 0,5-0,8mm compr., arqueado quando úmido, fortemente assimétrico; margem do filídio tinamente serrilhada $d a$ metade para cima; costa forte, mais próxima do lado côncavo, percurrente; células quadráticoarredondadas (9) 10-11(12) $\mu \mathrm{m}$; paredes pelúci das com uma papila em cada céluia com disposição oposta característica. Esporófito não examinacio.

\section{DISTRIBUIÇÃo GEOGRÁFICA}

Espécimes em Literatura: Brasil - (Mueller, 1901), como Mniomalia bernouillii, Bartram (1949), Steere (1948) e Mueller (1901)). Amazonas: região amazônica (Brotherus, 1909 e 1924); rio Negro, São Gabriel, col. R. Spruce 555 (Mueller, 1874) e Mitten (1869), ambos como Drepanophyllum viride). Pará: rio Amazonas, col. R. Spruce 553, 554 (Spruce (1867) e Mitten (1869), ambos como Drepanophyllum viride) .

Espécimes Examinados: Amazonas: Manaus Caracarai road, km 60, Reserva Campina, tronco podre, úmicio, col. D. Griffin, D.M. Vital \& O. Yano 563 pp. e 562, 1-VIII-1974 (INPA, FLAS, SP125463, SP125460). Mato Grossc: Barra do Garças, Acampamento dos Ingleses, ca $3 \mathrm{~km}$ SE $\left(12^{\circ} 51^{\prime} \mathrm{S}, 51945^{\prime} \mathrm{W}\right)$, sobre pau podre, mata de galeria, col. D.M. Vital 1399, 1-VI-1968, det. D.M. Vital (SP89834).

\section{Comentários}

Os hábitos de Phyllodrepanium e Mniomalia são muito semelhantes, isto é, crescem sobre tronco de árvore em decomposição. que ficam nos charcos próximos aos rios, igarapés, isto é, onde a umidade é alta e, geralmente, em matas fechadas e sombrias, às vezes de difícil acesso.

O gênero Mniomalia possui três espécies. senclo $M$. viridis, a única espécie brasileira, enquanto Phyllcdrepanium é monotípico $\operatorname{ccm} P$. falcifolium, ocorrendo no Brasil. 


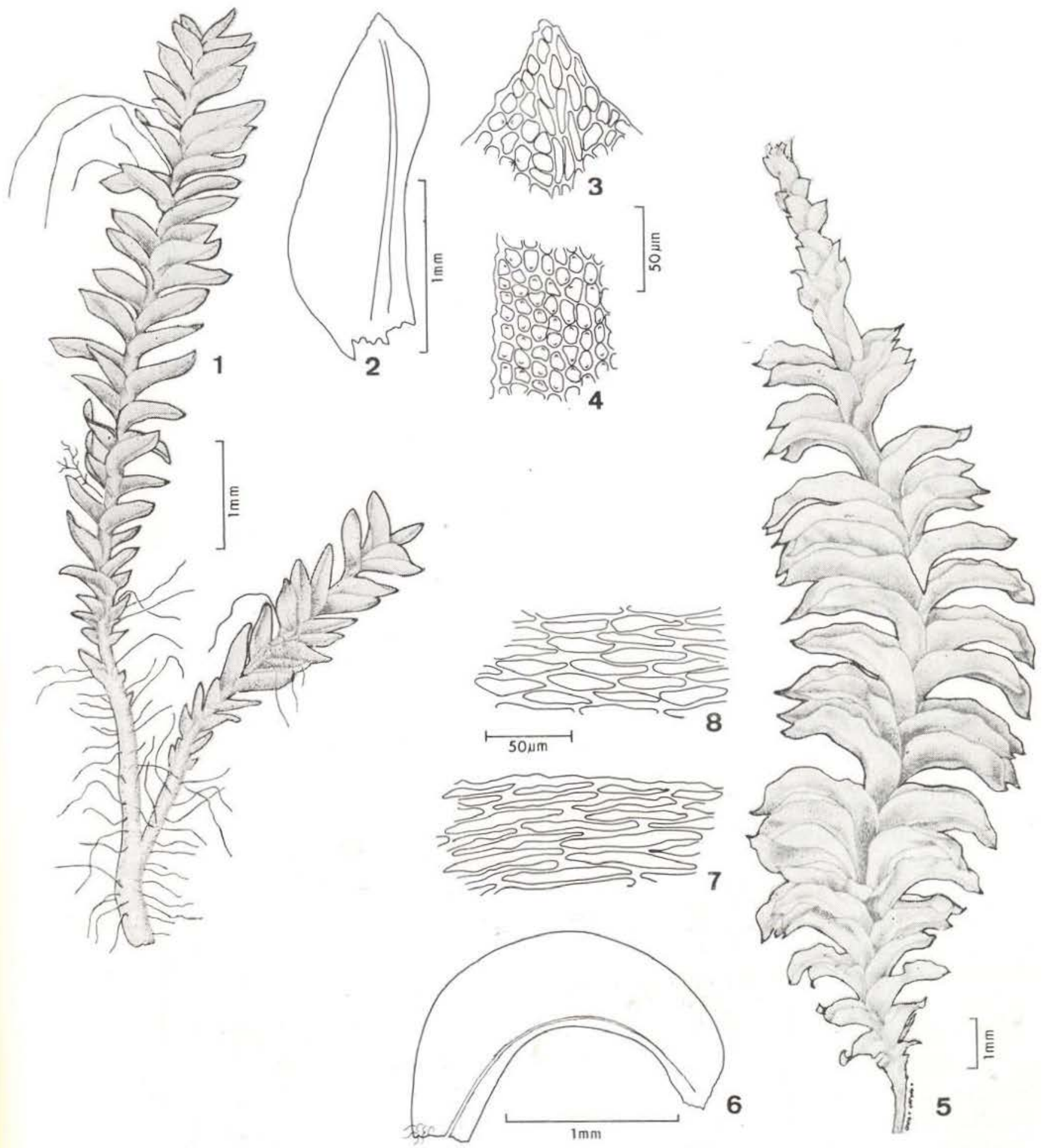

Fig. $1-4$ - Mniomalia viridis (Mitt.) C. Muell.: 1) Aspecto geral do gametófito; 2) Filídio; 3) Células do ápice do filídio; 4) Células medianas do filidio. Fig. 5-8 - Phyllodrepanium falcifolium (Schwaegr.) Crosby: 5) Aspecto geral do gametófito; 6) Filidio; 7) Células marginais do filidio; 8) Células medianas do filídio. 


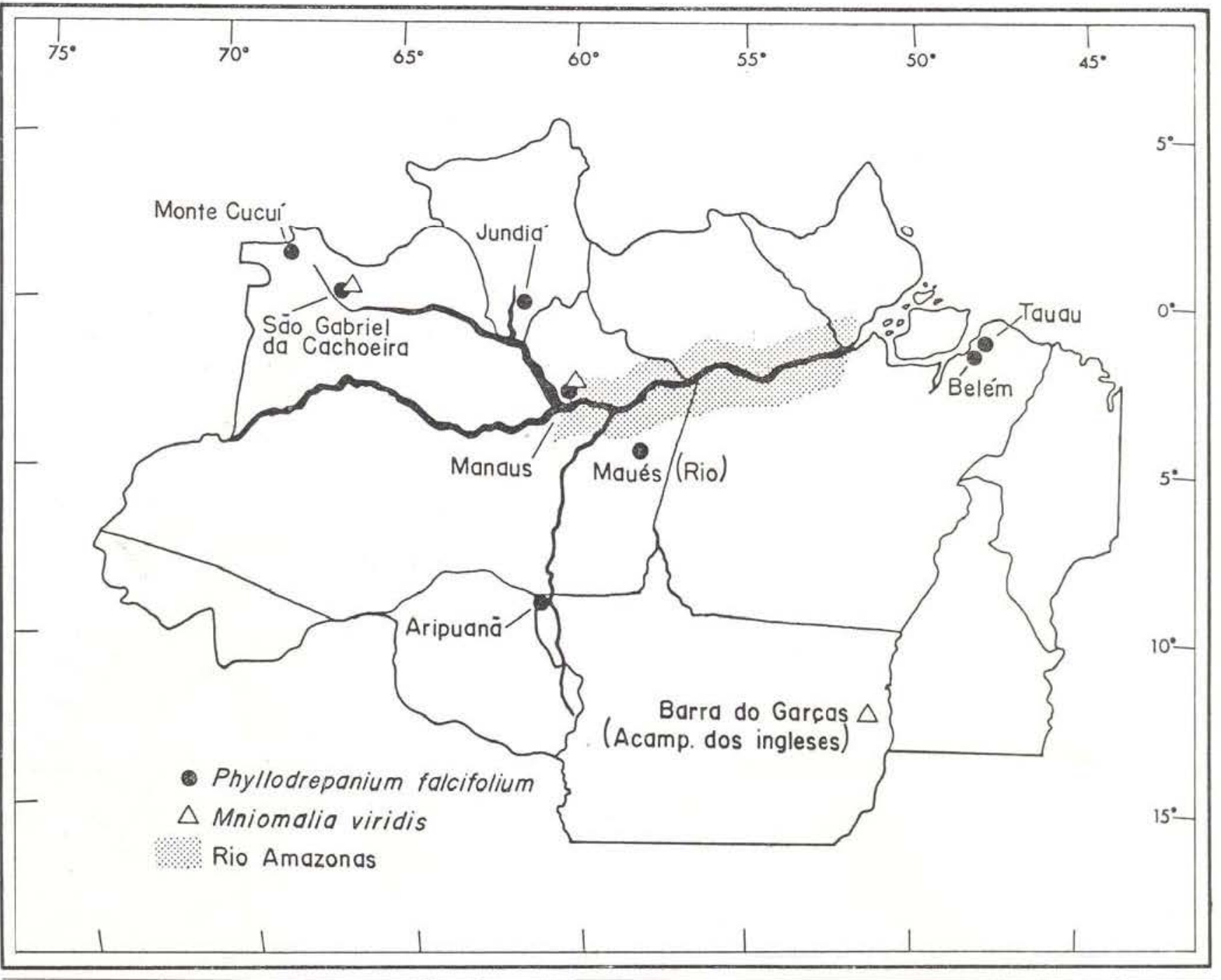

Fig. 9 - Distribuição geográfica de Phyllodrepanium falcifolium e Mniomalia viridis.

Até o momento, as espécies desta família foram encontradas ocorrendo somente na regiấc amazônica (Fig. 9) .

Fora do Brasil, a espécie Phyllodrepanium falcifolium é também encontrada em Trinidad, Honduras, Panamá. Hispaniola, São Domingos, Colômbıa, Venezuela, Guiana, Suriname, Guiana Francesa e Equador; a espécie Mniomalia viriclis ocorre na Guatemala e Equador.

O gênero Phyllodrepanium possui filídios falcados, com uma das lâminas bastante estreitadas e com células diferenciadas e mais alongadas na margem. No gênero Mniomalia, embora uma das lâminas seja igualmente estreitada, os filídios não são falcados, assim como não apresentam células marginais diferenciadas, assemelhando-se dessa forma ao gênero Fissidens .
Segundo Crosby (1970), os filídios menores de $P$. falcifolium apresentam células marginais bem diferenciadas e nas maiores a diferença é pequena. Nas exsicatas, a diferença observada foi muito pequena, tanto nos filídios menores como nos maiores; a costa não é tão excurrente como mostram as fig. 2-11 do autor citado.

A espécie $M$. viridis possui uma papila em cada célula com disposição oposta caracterís. tica pelo menos nos $3 / 4$ do filídio (base para o ápice do filidio).

\section{SUMMMARY}

A review of the Phyllodrepaniaceae (Eubryales, Bryopsida), as pertains to Brazil, is presented. Included are descriptions of the 2 species known to occur in Brazil, Phyllodrepanium falcifolium (Schwaegr.) Crosby 
and Mniomalia viridis (Mitt.) C. Muell., along with illustrations, distribution within the country (Amazonian Region), citations for specimens at IAN, INPA, MG and SP herbaria and from the literature.

\section{REFERENCIAS BIBLIOGRÁFICAS}

BARTRAM, Edwin B.

1949 - Mosses of Guatemala. Fieldiana Bot., 25: 1-442, fig. 1-190.

BROTHERUS, Viktor F.

1909 - Andreaceae und Bryales. In: Engler \& Prantl, Die Natürlichen Pflanzenfamilien. Leipzig: von Wilheln, série 1, 1(3): 265-268, 277-1246, fig. $162-165,179-861$.

1924 - Bryales. In: Engler \& Prantl, Natürlichen Pflanzenfamilien. Leipzig: von Wilheln, sér. 2, vol. 10 (1): 143-478, fig. 116-420.

CROSBY, Marshall R.

1970 - Some remarks on the genus Drepanophyllum Schwaegr. Revue Bryol. Lichen., 37 (2): $345-353$, fig. 2-11.

HORNSCHUC, Christianus F.

1840 - Musci. In: C.F.P. Martius, Flora Brasiliensis. Monachii, 1 (2): 1-99, pl. 1-4.
LISBOA, Regina C.L. \& LISBOA, Pedro L.B.

1978 - Contribuição ao conhecimento da flora do Aripuanã (Mato Grosso) II. Musci. Acta Amazonica, 8 (2): 143-148.

MITTEN, William

1869 - Musci austro-americani, enumeratio muscorum omnium austro-americanorum auctori hucusque. J. Linn. Soc. Bot., 12: 1-659.

MUELLER, Carl

1874 - Musci polynesiaci praesertium Vitiani et Somoani Graeffeani. J. Mus. Godeffroy 3 (6): $51-90$

1901 - Genera Muscorum Frondosorum. Leipzig: Eduard Kummer ed., 1-vii + 1-474.

SPRUCE, Richard

1867 - Catalogus muscorum fere omnium quos in Terris Amazonicis et Andinis, per annos 1849-1860. London, 1-22.

STEERE, William C.

1948 - Contribution to the bryogeography of Ecuador 1: a review of of the species of Musci preciously reported. Bryologist, 51 (3): 65167.

(Aceito para publicação em 22/01/81) 